\title{
Giant Right Coronary Artery Aneurysm Resembling Cardiac Tumor: A Case Report
}

\author{
Hongxia Wang, MD, Zhelan Zheng, PhD, Yun Mou, PhD \\ Echocardiography and Vascular Ultrasound Center, The First Affiliated Hospital, College of Medicine, Zhejiang University, \\ Hangzhou, China
}

\section{ABSTRACT}

Background: Coronary artery aneurysms are not uncommon vascular anomalies, but giant coronary artery aneurysms mimicking cardiac tumors are extremely rare and easily misdiagnosed as tumors. The management and prognosis are not well defined.

Case report: We describe a case of a 50-year-old woman, who had a transthoracic echocardiogram that suggested a cardiac tumor in the right ventricle adjacent to the tricuspid valve and without ventricular wall akinesia. Her medical history was unremarkable, and she had no obvious symptoms. A transesophageal echocardiogram and computed tomography angiogram examination suggested the tumor-like structure was a giant coronary aneurysm, originating from the distal portion of right coronary artery. The patient underwent surgical intervention and recovered uneventfully. Histopathology of the excised aneurysm showed fibrous tissue hyperplasia with collagenization and calcification in the wall of the artery.

Conclusion: Giant coronary artery aneurysms resembling heart tumors are extremely rare. Differentiating them from cardiac tumors can sometimes be difficult. This rare case emphasizes the importance of combining multiple imaging methods, which helps make accurate diagnosis. For treatment, surgical intervention may be the appropriate therapeutic strategy for the rare lesion.

\section{INTRODUCTION}

Coronary artery aneurysm (CAA) is an abnormal dilatation of a localized segment of the coronary artery. The rate of this type of aneurysm is reported to be $1 \%$ to $4 \%$ of coronary angiography findings [Syed 1997]. However, a giant coronary aneurysm mimicking a heart tumor is relatively rare. Although the aneurysm often is diagnosed by cardiac imaging, the diagnosis still be difficult sometimes, especially for the unusual coronary aneurysm mimicking a heart tumor. We present here a rare patient, who initially was suspected of having a cardiac tumor in the right ventricle by transthoracic echocardiography

Received December 23, 2019; accepted Fanuary 30, 2020.

Correspondence: Hongxia Wang, Echocardiography and Vascular Ultrasound Center, The First Affiliated Hospital, College of Medicine, Zhejiang University, Hangzhou, 310003, China; +86-0571-87236516 (e-mail: 1505113@zju.edu.cn).
(TTE). An additional examination showed that the tumor-like mass was a giant right CAA. The pathophysiology of CAA is not completely understood. Treatment and prognosis of a

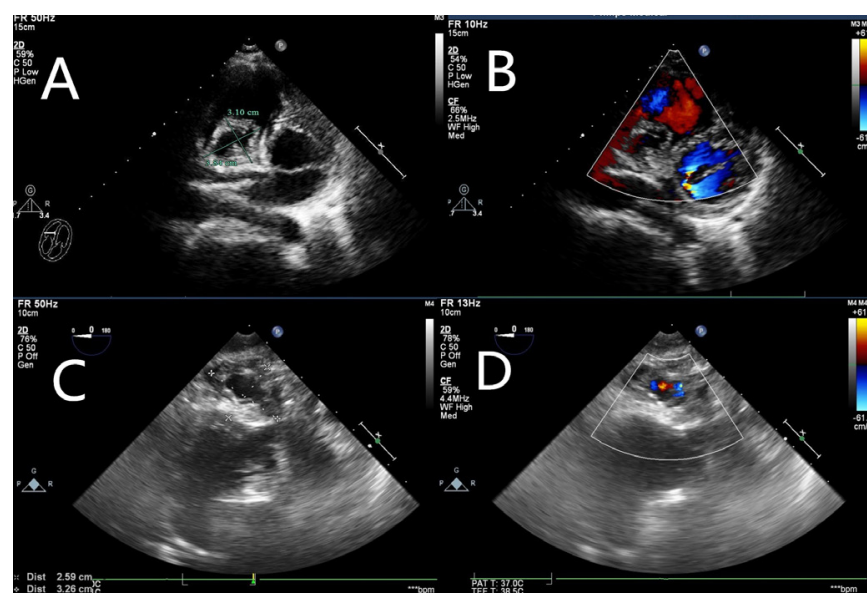

Figure 1. A, B, TTE shows a giant right CAA presenting as a right ventricular mass $(3.8 \times 3.1 \mathrm{~cm})$. C, TEE shows a $3.3 \times 2.6-\mathrm{cm}$ solid-cystic structure. $\mathrm{D}$, On color doppler, blood flow is seen in the cystic structure.

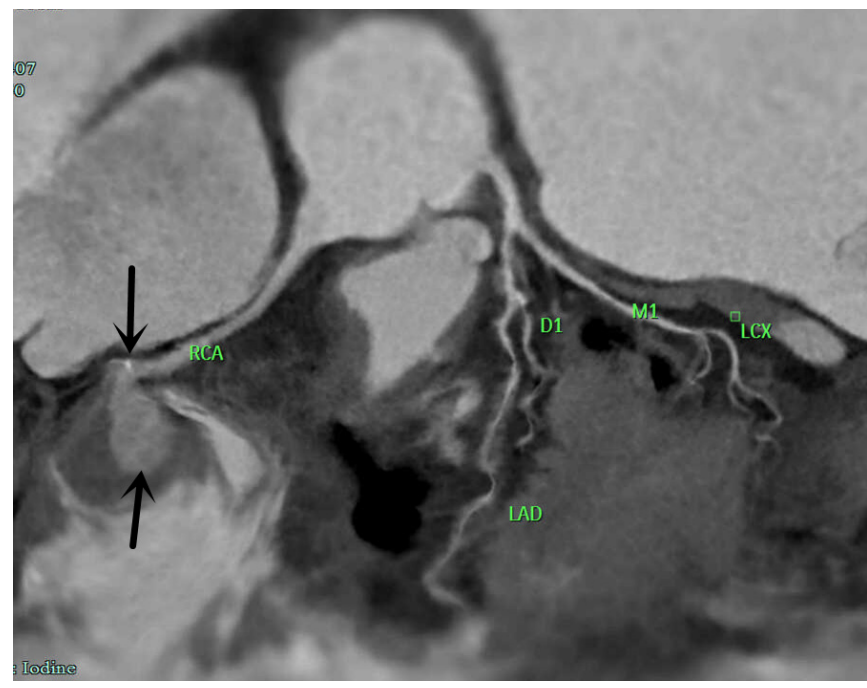

Figure 2. CTA shows a slightly dilated right coronary artery, and CAA located near the distal portion of the right coronary artery (black arrow). RCA: right coronary artery. 


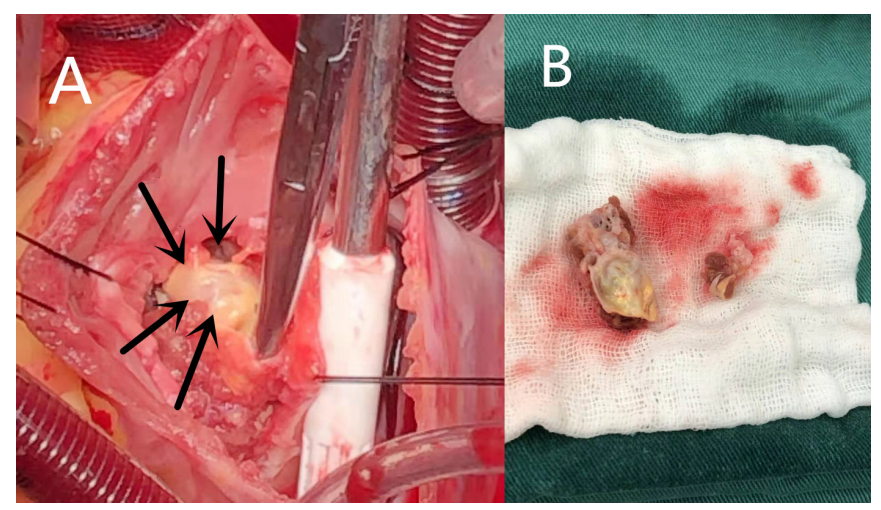

Figure 3. A, Intraoperative photograph shows the appearance of the CAA (black arrows). B, Photograph of the excised specimen shows a coarse coronary artery intima and there is no thrombus in the cavity.

coronary aneurysm are controversial. We discuss this unusual disease for better awareness and understanding.

\section{CASE REPORT}

A 50-year-old woman was admitted to our hospital with the diagnosis of a cardiac tumor. Her medical history was unremarkable, and she had no obvious symptoms. She did not smoke. Her blood pressure and heart rate were normal. Laboratory tests showed normal levels of triglycerides and cholesterol. Antinuclear antibody was negative, and the serum immunoglobulin 4 level was within the normal range. Electrocardiography showed a low-flat $\mathrm{T}$ wave. Diseases potentially relevant for development of an aneurysm were not found in the patient's family history. An echocardiographic examination showed a tumor in the right ventricle. TTE showed a $38 \times 31 \mathrm{~mm}$ regular mass with a clear external border in the right ventricle adjacent to the posterior leaflet of the tricuspid valve. The mass was cystic in the center, solid in the periphery, and color doppler showed no obvious blood flow in the sac (Figure $1 \mathrm{~A}$ and $\mathrm{B}$ ).

Tricuspid valvular leaflets were normal and had no obvious regurgitation. Because of the regular shape of this mass, the distinct external border, and absence of pericardial effusion, a benign tumor was considered. Transesophageal echocardiography (TEE) showed that the surrounding echo of mass was slightly high and similar to the myocardial echo, and on color doppler blood flow signals were observed in the central sac. (Figure $1 \mathrm{C}$ and D) Subsequently, computed tomography angiogram (CTA) showed a slightly dilated right coronary artery and an enhanced mass originating from the distal portion of the right coronary artery (Figure 2).

The patient underwent a surgical operation. The tumor was located in the right ventricle below the posterior tricuspid valve. The posterior leaflet of the tricuspid valve was cut open, and the tumor was completely exposed. The surface of the tumor was covered by myocardial tissue. The muscle tissue was separated, and below it, a dilated vascular structure was observed (Figure 3).

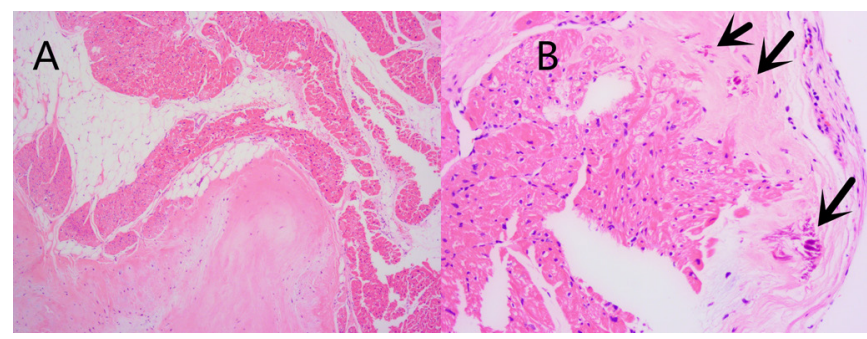

Figure 4. Pathological manifestations as shown by hematoxylin-eosin staining. A, Histological findings show fibrous tissue hyperplasia with accumulation of collagen in the media of the artery wall (magnification, $\times 50$ ); $B$, Calcification in the artery wall (black arrow) (magnification, $\times 200$ ).

This vascular structure communicated with the right coronary artery. Therefore, the mass was confirmed as a giant coronary aneurysm protruding into the right ventricular cavity along with the surface myocardium, and it resembled a tumor. The surgeon resected the aneurysm and closed the stump of the feeding vessel by continuous suture with 5-0 prolene.

The inside view of the excised specimen of the aneurysm showed a coarse coronary artery intima, and there was no thrombus in the cavity. Histopathology of the aneurysm showed fibrous tissue hyperplasia with accumulation of collagen and calcification in the media of the artery (Figure 4 $\mathrm{A}$ and $\mathrm{B})$.

The patient recovered well and was discharged 9 days later. Informed consent for publication of clinical data was obtained from the study participant.

\section{DISCUSSION}

A CAA is defined as localized coronary dilation, which exceeds the adjacent normal segment by $50 \%$. However, a giant aneurysm is termed as larger than $2 \mathrm{~cm}$ and is unusual. Most CAAs are associated with atherosclerosis and Kawasaki disease [Ouali 2011]. Other causes of CAAs include Marfan's syndrome, connective tissue diseases, arteritis [Pahlavan 2006], and IgG4-related coronary periarteritis [Ikutomi 2011]. Recently, an increasing amount of reports have suggested that implantation of drug-eluting stents causes coronary aneurysms [Ahn 2010]. The pathophysiology of CAA still remains unclear, but the coronary artery segment can progressively dilate with destruction of the arterial media, thinning of the arterial wall, and increased wall stress. In our case, the histopathological changes showed fibrous tissue hyperplasia, accumulation of collagen, and calcification in the media of the artery.

Patients' clinical features vary according to the location, size of the CAA, and degree of coronary stenosis. The majority of patients are asymptomatic and may present with angina pectoris, myocardial infarction, heart failure, and thrombotic disease [Abou 2017]. So, coronary artery aneurysms have no specific symptoms and signs. In our case, the patient was asymptomatic and was observed accidentally on echocardiography. 
The standard of reference for the diagnosis of CAA is coronary angiography, which provides the location, size, shape, and coexisting abnormalities, such as coronary artery stenosis or blocking [Pahlavan 2006]. However, coronary angiography is invasive, and the true size of the coronary aneurysm sometimes can be underestimated. Echocardiography and CTA play an important role in diagnosing CAAs because of their rapid, non-invasive, and reliable characteristics.

Echocardiography clearly can show the inner structure of the CAA, valvular situation, and presence or absence of a thrombus, but has the disadvantage of not being able to show the whole stage of the coronary artery. CTA has the advantage of showing the whole course of the coronary artery and blood flow. However, CTA has a limitation in showing thrombi inside of vessels. The contribution of magnetic resonance imaging (MRI) is its excellent soft tissue contrast, which can provide accurate information about tumoral tissue characteristics.

In our case, TTE showed a mass in the right ventricle, but did not show blood flow in the mass. TEE showed a blood flow signal in the sac of the mass because of its high resolution. CTA clearly showed an enhanced mass communicating with the right coronary artery. After a variety of examinations, the aneurysm finally was diagnosed. The giant coronary aneurysm mimicking cardiac tumor is rare and only a few cases have been reported. Vivek et al presented a mass measuring $2.4 \times 2.7 \mathrm{~cm}$ in the right atrium on transthoracic echocardiogram after right coronary artery stent implantation [Vivek 2014]. At last, coronary angiogram confirmed that the mass was a giant aneurysm. Małgorzata et al reported a case of suggesting cardiac tumor on echocardiography [Małgorzata 2013]. However, MRI examination revealed a suspected coronary aneurysm, and CTA confirmed it right CAA. Therefore, a coronary aneurysm is the main differential diagnosis for a cardiac tumor and accurate diagnosis depends on multiple images.

Management of CAAs consists of surgical intervention, medical treatment, and stent implantation. Surgical intervention generally is recommended as the preferred treatment method for giant CAAs [Jha 2009]. Ligation of aneurysms with distal bypass grafting plication and repair of the aneurysm are common surgical methods. Medical treatment for CAAs includes antiplatelet or antithrombotic therapy for reducing the risk of thrombus. Percutaneous stent implants currently are available to treat selected patients. However, long-term outcomes of these stents still are unknown
[Zeb 2012]. In our case, the surgeon removed the aneurysm and closed the stump of the feeding vessel, without coronary artery bypass grafting because the patient had no evidence of myocardial ischemia.

\section{CONCLUSION}

We present a rare case with a giant CAA resembling a heart tumor. Differentiating it from cardiac tumors sometimes can be difficult. This rare case emphasizes the importance of combining multiple imaging methods to help make an accurate diagnosis. For treatment, surgical intervention may be the appropriate therapeutic strategy for rare giant CAAs.

\section{REFERENCES}

Abou Sherif S, Ozden Tok O, Tassköylü Ö, et al. 2017. Coronary artery aneurysms: a review of the epidemiology, pathophysiology, diagnosis, and treatment. Front Cardiovasc Med 4:24.

Ahn CM, Hong BK, Kim JY, et al. 2010. Incidence and natural history of coronary artery aneurysm developing after drug-eluting stent implantation. Am Heart J 160: 987-994.

Ikutomi M, Matsumura T, Iwata H, et al. 2011. Giant tumorous legions surrounding the right coronary artery associated with immunoglobulinG4-related systemic disease. Cardiology 120: 22-26.

Jha NK, Ouda HZ, Khan JA, Eising GP, Augustin N. 2009. Giant right coronary artery aneurysm--case report and literature review. J Cardiothorac Surg 4:18.

Małgorzata UZ,Robert PB,Maciej K. 2013. Right coronary artery aneurysms in patient with suspicion of heart tumor. Przegl Lek 70(5):356-8.

Ouali S, Kacem S, Ben Fradj F, Gribaa R, Naffeti E, Remedi F, et al. 2011. Takayasu arteritis with coronary aneurysms causing acute myocardial infarction in a young man. Tex Heart Inst J 38: 183-186.

Pahlavan PS, Niroomand F. 2006. Coronary artery aneurysm: a review. Clin Cardiol 29:439-43.

Syed M, Lesch M. 1997. Coronary artery aneurysm: a review. Prog Cardiovasc Dis 40:77-84.

Vivek M, Muhammad AH, Nagesh SA, et al. 2014. Giant coronary aneurysm presenting as a cardiac mass on transthoracic echocardiogram. BMJ Case Rep doi:10.1136/bcr-2013-202536.

Zeb M, McKenzie DB, Scott PA, Talwar S. 2012. Treatment of coronary aneurysms with covered stents: a review with illustrated case. J Invasive Cardiol 24(9):465-9. 